\title{
Composição e diversidade de Scarabaeoidea (Coleoptera) atraídos por armadilha de luz em área rural no norte do Paraná, Brasil
}

\author{
Daniele C. Ronqui ${ }^{1} \&$ José Lopes $^{2}$
}

1. Bacharelanda em Ciências Biológicas da Universidade Estadual de Londrina. (DanieleR@fundacaoboticario.org.br)

2. Departamento de Biologia Animal e Vegetal, Universidade Estadual de Londrina, Campus Universitário, 86051-990 Londrina, PR, Brasil. (jea@uel.br)

\begin{abstract}
Composition and diversity of Scarabaeoidea (Coleoptera) attracted by light trap in the rural areas of Northern Paraná. The most devastated environments in the State of Paraná are those with great agricultural potential, and most human interference actions on the environment are seldom preceded by a careful study of the fauna, which would reveal the diversity and abundance of animals in these areas. This study aimed to describe the Scarabaeoidea fauna attracted by light traps in rural areas of the Tamarana County, Paraná, Brazil, and to contribute to the ecological knowledge on species of this group. The sampling was carried out quarterly, from March 2002 to April 2003, in two rural properties, using a modified "Luiz de Queiroz" light trap. The light traps captured 2447 specimens, distributed into 10 families, 24 genera and 67 species. The three most abundant species were also the most frequent: Aphodius lividus (Olivier, 1789), Melolonthidae sp. 1 e Ataenius sp. 5. The highest A. lividus population density occurred in April, fall season, whereas Melolonthidae sp. 1 and Ataeucus were most frequently found in Octubre and November, spring. The majority of the species were represented by few individuals $\left(H^{\prime}=1,74\right)$, and the distribution of individuals species was relatively uniform $\left(\mathrm{J}^{\prime}=0,95 ; \mathrm{S}=0,20\right)$. A large number of families was recorded, and they were represented by few individuals. There was a predominance of families of detritivores - Aphodiidae, Scarabaeidae, Hybosoridae and phytophagus - Melolonthidae, Dynastidae and Rutilidae. The study collected 25 detritivore species among the 1422 specimen recorded.
\end{abstract}

KEYWORDS. Scarabaeidae, diversity, rural area, light trap.

RESUMO. No Estado do Paraná, as áreas mais devastadas são aquelas com potencial agrícola e as ações de inferência no meio raramente são precedidas do estudo faunístico que permitam avaliar a diversidade e abundância dos animais das áreas atingidas. Este trabalho objetivou catalogar a escarabeoideofauna atraída por armadilha de luz na área rural do município de Tamarana, Paraná e contribuir com o conhecimento ecológico de espécies deste grupo. As coletas foram realizadas em duas propriedades, utilizando armadilha de luz modelo Luiz de Queiroz modificada. Estas tiveram periodicidade quinzenal de março de 2002 a abril de 2001 . Foram capturados 2.447 espécimens, distribuídos em 10 famílias, 24 gêneros e 67 espécies. As três espécies mais abundantes também foram as mais freqüentes: Aphodius lividus (Olivier, 1789), Melolonthidae sp. 1 e Ataenius sp. 5. A maior abundância de A. lividus ocorreu no outono, durante o mês de abril, enquanto que Melolonthidae sp. 1 e Ataeucus sp. 5 foram mais abundantes em outubro e novembro. A maioria das espécies foram representadas por poucos indivíduos $\left(H^{\prime}=1,74\right)$ e as espécies apareceram de maneira uniforme $\left(J^{\prime}=0,95\right.$; $\mathrm{S}=0,20)$. Ocorreu um maior número de famílias que estavam representadas por poucos indivíduos. Houve predominância das famílias que apresentam hábitos alimentares detritívoros - Aphodiidae, Scarabaeidae, Hybosoridae e fitófagos - Melolonthidae, Dynastidae e Rutelidae. Para os grupos de hábito detritívoro, foram coletados 25 espécies, num total de 1.422 espécimes.

PALAVRAS-CHAVE. Scarabaeidae, diversidade, área rural, armadilha de luz.

O Estado do Paraná sofreu uma grande devastação de sua cobertura vegetal nativa e, atualmente, apresentase apenas com alguns fragmentos remanescentes de florestas circundadas por terras cultivadas ou áreas de pastagens (MEDRi \& Lopes, 2001a). As condições ambientais em uma floresta são bastante diferentes das de uma área aberta; a destruição dos ambientes naturais para a criação de pastagem ou monoculturas provoca alterações nos fatores abióticos, ocasionando mudança na estrutura e composição das comunidades, que podem sofrer perda de espécies não-adaptadas às novas condições ambientais (MEDRI \& LOPES, 2001b).

Dificilmente as devastações são precedidas, ou mesmo sucedidas, de estudos faunísticos que permitam avaliar a composição, diversidade e abundância dos animais das áreas atingidas (MARINONI \& DUTRA, 1991). Visando amenizar este problema inventarial, torna-se importante o estudo da fauna de áreas ainda preservadas e de áreas degradadas, para estudos comparativos e melhor compreensão do funcionamento das comunidades e ecossistemas.
Quando há um desequilíbrio na natureza relacionado à degradação ambiental, os animais que mais causam problemas ao homem são os insetos (MARINONI \& Dutra, 1991); por isso, o conhecimento do comportamento das espécies torna o controle, quando necessário, mais racional (CIVIDANES et al., 1980).

Os escarabeídeos (Coleoptera) participam deste complexo contexto que envolve as alterações antrópicas ambientais. O grupo está representado por aproximadamente 20 mil espécies, das quais muitas são coprófagas e atuam como importantes agentes na reciclagem de fezes de mamíferos, especialmente dos grandes herbívoros. A maioria é herbívora na fase larval e necrófaga quando adultos ou alimentam-se apenas de pólen e néctar. Poucas são parasitas.

As espécies de escarabeídeos são ativas durante o dia ou no período noturno, mas algumas podem exercer atividade tanto durante à noite quanto no período diurno (HALFFTER \& Edmonds, 1982). Desta forma, armadilhas luminosas podem ser usadas como ferramenta para levantamento deste grupo de besouros. O uso deste tipo 
de armadilha no levantamento de populações de insetos constitui uma prática freqüente (CIVIDANES et al., 1980), pois atraem insetos fototácticos com atividade noturna (MARINONI \& DUTRA, 1991) e constituem um método usado para monitoramento geral e temporal da abundância de insetos (KATO et al., 2000).

NATH \& Singh (1994) fizeram uso de armadilha de luz para monitorar a emergência e abundância de escarabeídeos na Índia. Flechtmann et al. (1995) utilizaram este mesmo equipamento para estudo de espécies de Scarabaeidae coprófagos. Outros conhecimentos ecológicos sobre este grupo de insetos foram obtidos por KATO et al. (2000), em estudo de oito espécies de Scarabaeidae na Malásia.

Este trabalho teve por objetivo levantar a escarabeoideofauna atraída por armadilha de luz em uma região de pastagem com pequenos fragmentos de mata no município de Tamarana, Paraná, Brasil e estabelecer parâmetros relacionados à estrutura de comunidade, sua diversidade e dinâmica temporal.

\section{MATERIAL E MÉTODOS}

A área de estudo situa-se no terceiro Planalto Paranaense, na porção centro-norte do Estado do Paraná, município de Tamarana $\left(25^{\circ} 27^{\prime} \mathrm{S} ; 51^{\circ} 15^{\prime} \mathrm{W}\right)$, distante cerca de 57 km da cidade de Londrina, Paraná. A região encontrase a aproximadamente $740 \mathrm{~m}$ de altitude e sua vegetação primitiva, classificada fitogeograficamente como Floresta Estacional Semidecidual, foi totalmente dizimada, sendo substituída principalmente por pastagem, embora se constate algum espaço ocupado por agricultura de pequeno porte. Corresponde a uma paisagem de colina, restando pequenas manchas de mata secundária nas glebas e mata ciliar ao longo dos ribeirões, cujas bacias hidrográficas são bastante ricas em ramificações aquíferas (Fig. 1).

O clima da região, conforme MAACK (1981) e segundo a classificação de Köppen, é do tipo Cfa, ou seja, tropical úmido com verões quentes e invernos com geadas pouco freqüentes e sem estação seca definida. A média pluviométrica anual é de $1.714,7 \mathrm{~mm}$ e a temperatura média anual é de $21^{\circ} \mathrm{C}$. O solo da região é classificado como Terra Roxa Estruturada Eutrófica, sendo predominantemente argiloso, profundo, com boa

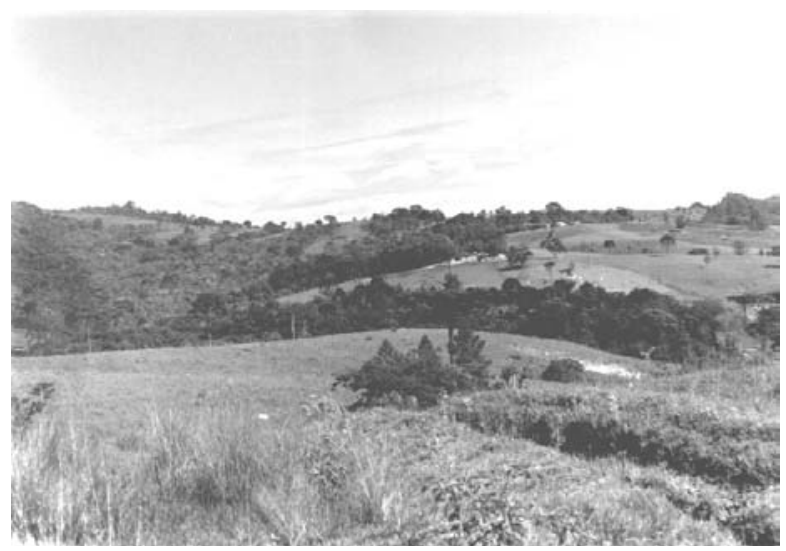

Fig. 1. Vista geral da área de estudo em Tamarana, Paraná. porosidade, drenagem e coloração arroxeada (EMBRAPA, 1984; URBAN, 1996).

Foram utilizadas para as coletas duas armadilhas de luz modelo "Luiz de Queiroz" modificada, como réplica, já que os ambientes eram semelhantes. Estas armadilhas foram instaladas em duas propriedades, distantes aproximadamente três quilômetros entre si, presas ao galho de uma árvore, ficando suspensas a dois metros do solo.

As coletas ocorreram de março de 2000 a abril de 2001, quando as armadilhas permaneceram ligadas por uma noite, das $18 \mathrm{~h}$ as $6 \mathrm{~h}$, a cada 15 dias. A armadilha de número um foi instalada em uma árvore localizada no final de uma área com plantas frutíferas, eucaliptos e grevilhas. Logo abaixo situa-se um corredor da área de pastagens com aproximadamente 100 metros de largura, seguido de mata secundária que circunda um pequeno ribeirão. A armadilha número dois foi instalada em pomar de citrus, próxima à residência do sitiante e circundada por pastagem. Esta armadilha poderia estar sendo influenciada pela iluminação doméstica.

Os escarabeóideos foram separados por morfoespécies e representantes de cada uma destas foram enviados a especialista para identificação. O restante dos espécimes coletados foram identificados por comparação e o material estudado está depositado no Museu de Zoologia da Universidade Estadual de Londrina (MZUEL), Londrina, Paraná.

Com a finalidade de avaliar a diversidade das espécies coletadas de Scarabaeoidea, foi utilizado o índice de Shannon $\left(\mathrm{H}^{\prime}=-\Sigma\right.$ pi.log pii) e para avaliar a uniformidade da distribuição de indivíduos por espécies coletadas, aplicou-se o índice de Pielou ( $\left.\mathrm{J}^{\prime}=\mathrm{H}^{\prime} / \log \mathrm{S}\right)$. Como complementaridade aplicou-se o índice de dominância de espécie de Simpson $\left(\mathrm{D}=\Sigma p i^{2}\right)$. Os índices foram calculados por meio do programa Past (HAMmer et al., 2004).

Constatada a diversidade de Scarabaeoidea capturada e a flutuação quantitativa de seus representantes, optou-se pela criação de classes de abundância para facilitar a análise, estabelecendo-se as abrangências destas classes conforme perfil quantitativo para cada táxon .

Adotou-se neste trabalho a classificação de Воотн et al. (1990) para Scarabaeoidea.

\section{RESULTADOS}

Nas 28 coletas realizadas entre o período de março de 2000 a abril de 2001, foram capturados 2.447 espécimes, distribuídos em 67 espécies, 24 gêneros e 10 famílias (Tab. I). A armadilha luminosa utilizada mostrou-se atrativa para os Scarabaeoidea das famílias Aphodiidae (1.370 indivíduos de 16 espécies) e Melolonthidae (796 indivíduos de 11 espécies). Embora Dynastidae tenha sido representada por poucos indivíduos (86), o foi por ampla riqueza específica (16 espécies).

Do total de indivíduos coletados, as quatro espécies mais freqüentes e mais abundantes, foram Aphodius lividus (Olivier, 1789) (32,6\%), Melolonthidae sp. $1(29 \%)$, Ataenius sp. $5(8,3 \%)$ e Aphodius sp. 2 (4,5\%) (Tab. 1). A diversidade foi alta, mas apenas quatro espécies foram dominantes em relação à abundância.

Somando-se o coletado nas duas áreas, encontrou- 
Tabela I. Quantidade e representação porcentual para cada espécie de Scarabaeoidea coletada em armadilha de luz no município de Tamarana, Paraná, de março de 2000 a abril de 2001.

\begin{tabular}{|c|c|c|c|}
\hline Família & Espécies & Número de exemplares & $\%$ \\
\hline \multirow{16}{*}{ Aphodiidae } & Aphodius lividus (Olivier, 1789) & 796 & 32,5 \\
\hline & Aphodius sp.1 & 19 & 0,8 \\
\hline & Aphodius sp.2 & 111 & 4,5 \\
\hline & Aphodius sp.3 & 17 & 0,7 \\
\hline & Aphodius sp.4 & 7 & 0,3 \\
\hline & Aphodius sp.5 & 1 & 0,0 \\
\hline & Aphodius sp.6 & 7 & 0,3 \\
\hline & Aphodius sp.7 & 12 & 0,5 \\
\hline & Aphodius sp.8 & 8 & 0,3 \\
\hline & Aphodius nigrita (Fabricius, 1792) & 30 & 1,2 \\
\hline & Ataenius sp.1 & 11 & 0,4 \\
\hline & Ataenius sp.2 & 7 & 0,3 \\
\hline & Ataenius sp.3 & 61 & 2,5 \\
\hline & Ataenius sp.4 & 7 & 0,3 \\
\hline & Ataenius sp.5 & 203 & 8,3 \\
\hline & Platytomus sp. & 73 & 3,0 \\
\hline \multirow[t]{16}{*}{ Dynastidae } & Augoderia nitidula Burmeister, 1847 & 4 & 0,2 \\
\hline & Bothinus sp.1 & 15 & 0,6 \\
\hline & Bothinus sp.2 & 1 & 0,0 \\
\hline & Chalepides sp. & 3 & 0,1 \\
\hline & Cyclocephala sp.1 & 17 & 0,7 \\
\hline & Cyclocephala sp. 2 & 18 & 0,7 \\
\hline & Cyclocephala sp.3 & 5 & 0,2 \\
\hline & Cyclocephala sp.4 & 2 & 0,1 \\
\hline & Cyclocephala sp.5 & 2 & 0,1 \\
\hline & Cyclocephala sp. 6 & 2 & 0,1 \\
\hline & Cyclocephala sp.7 & 9 & 0,4 \\
\hline & Cyclocephala sp. 8 & 1 & 0,0 \\
\hline & Cyclocephala sp.9 & 3 & 0,1 \\
\hline & Stenocrates sp. & 2 & 0,1 \\
\hline & Strategus surinamensis (Burmeister, 1847) & 1 & 0,0 \\
\hline & Strategus validus (Fabricius, 1775) & 1 & 0,0 \\
\hline Ceratocanthidae & Germarostes macleayi (Perty, 1830) & 1 & 0,0 \\
\hline \multirow{11}{*}{ Melolonthidae } & Melolonthidae sp. 1 & 708 & 28,9 \\
\hline & Melolonthidae sp. 2 & 1 & 0,0 \\
\hline & Melolonthidae sp.3 & 28 & 1,1 \\
\hline & Melolonthidae sp.4 & 7 & 0,3 \\
\hline & Melolonthidae sp.5 & 15 & 0,6 \\
\hline & Melolonthidae sp.6 & 12 & 0,5 \\
\hline & Melolonthidae sp.7 & 16 & 0,7 \\
\hline & Melolonthidae sp.8 & 4 & 0,2 \\
\hline & Melolonthidae sp.9 & 3 & 0,1 \\
\hline & Melolonthidae sp.10 & 1 & 0,0 \\
\hline & Phyllophaga sp. & 1 & 0,0 \\
\hline \multirow[t]{9}{*}{ Rutelidae } & Anomala sp.1 & 20 & 0,8 \\
\hline & Anomala sp. 2 & 8 & 0,3 \\
\hline & Anomala sp.3 & 1 & 0,0 \\
\hline & Geniates sp. & 13 & 0,5 \\
\hline & Geniatosoma sp. & 3 & 0,1 \\
\hline & Leucothyreus sp.1 & 25 & 1,0 \\
\hline & Leucothyreus sp.2 & 7 & 0,3 \\
\hline & Leucothyreus sp.3 & 1 & 0,0 \\
\hline & Trizogeniates sp. & 9 & 0,4 \\
\hline \multirow[t]{6}{*}{ Scarabaeidae } & Dichotomius boss (Blanchard, 1843) & 62 & 2,5 \\
\hline & Dichotomius carbonarius (Mannerhein, 1829) & 1 & 0,0 \\
\hline & Dichotomius fimbriatus (Harold, 1869) & 12 & 0,5 \\
\hline & Ontherus appendiculatus (Mannerhein, 1829) & 1 & 0,0 \\
\hline & Ontherus sulcator (Fabricius, 1775) & 2 & 0,1 \\
\hline & Uroxys prox dilaticollis & 1 & 0,0 \\
\hline \multirow[t]{2}{*}{ Ochodaeidae } & Parochodaeus sp. & 3 & 0,1 \\
\hline & Parochodaeus cornutus (Ohaus, 1910) & 1 & 0,0 \\
\hline \multirow[t]{3}{*}{ Hybosoridae } & Chaetodus sp.1 & 1 & 0,0 \\
\hline & Chaetodus sp.2 & 7 & 0,3 \\
\hline & Chaetodus prox. exaratus & 1 & 0,0 \\
\hline \multirow[t]{2}{*}{ Bolboceratidae } & Bolbapium sculpuratum (Mannerheim, 1829) & 3 & 0,1 \\
\hline & Neoathyreus brasilienzis (Howden, 1976) & 11 & 0,4 \\
\hline Trogidae & Polynoncus sp. & 1 & 0,0 \\
\hline Total & & 2447 & 100,0 \\
\hline
\end{tabular}


se maior abundância nos meses de outubro e novembro, na estação da primavera (Fig. 2).

Aphodius lividus apresentou maior abundância em abril, enquanto que a de Melolonthidae sp. 1 ocorreu em outubro e novembro e a de Ataenius sp. 5 em outubro, ambos na primavera (Fig. 2).

Embora os dois locais de coleta apresentem fisionomia de vegetação e topográfica semelhante, houve maior abundância de coleta no local um (1.678), quando comparado com o local dois (769), sendo 23 espécies coletadas com exclusividade no ponto um, 10 espécies no ponto dois e 34 espécies ocorreram em ambos os locais.

O índice de diversidade (H') da amostra foi de 1,74. Este valor pode estar influenciado pela ocorrência de muitas espécies coletadas serem representadas por poucos indivíduos. A relação do número de espécies com o número de indivíduos coletados comprovam o alto valor de diversidade obtido com o índice de Shannon. O índice de uniformidade ( $\mathrm{J}$ ') foi de 0,95 , o que mostra que as espécies ocorreram de maneira uniforme durante o período de estudo, não ocorrendo dominância de espécies. Isso também é comprovado pelo baixo índice de Simpson $(D=0,20)$ responsável por verificar o grau de dominância na comunidade.

A relação entre o número de indivíduos coletados e o número de famílias demonstrou que ocorreu maior número de famílias que estavam representadas por poucos indivíduos (Fig. 3). O mesmo ocorreu com as espécies, que também foram coletadas em grande número e representadas por poucos indivíduos (Fig. 4).

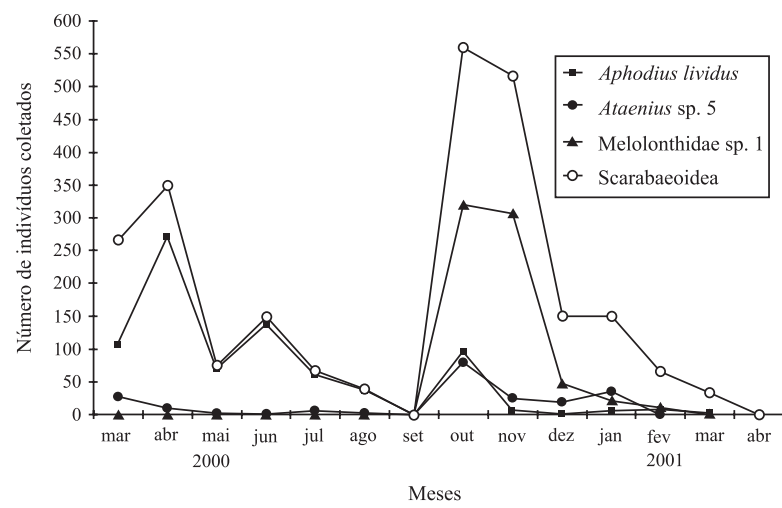

Fig. 2. Distribuição anual do total de Scarabaeoidea e das três espécies mais abundantes coletadas em armadilhas de luz no município de Tamarana, Paraná, de março de 2000 a abril de 2001.

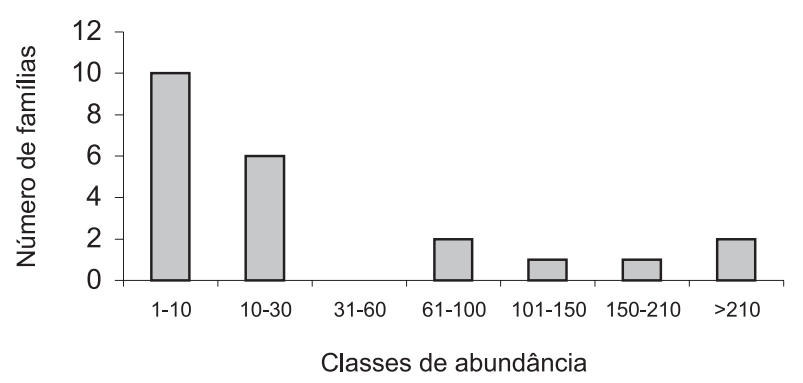

Fig. 3. Relação do número de indivíduos pelo número de famílias de Scarabaeoidea coletadas em armadilhas de luz no município de Tamarana, Paraná, de março de 2000 a abril de 2001.

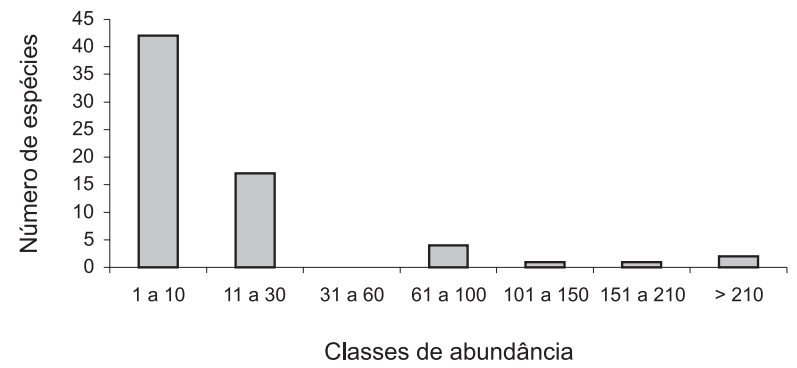

Fig. 4. Relação de indivíduos por espécies de Scarabeoidea coletadas em armadilhas de luz no município de Tamarana, Paraná, de março de 2000 a abril de 2001.

As espécies coletadas distribuem-se, basicamente, em famílias que apresentam hábitos alimentares detritívoros - Aphodiidae, Scarabaeidae, Hybosoridae e fitófagos - Melolonthidae, Dynastidae e Rutelidae. Para os grupos de hábito detritívoro, foram coletadas 25 espécies, num total de 1.422 exemplares com esse hábito alimentar.

\section{DISCUSSÃO}

De forma geral, a densidade populacional de Scarabaeoidea foi maior na primavera; esta pode ser estratégia de sobrevivência de insetos univoltinos. O adulto emerge no final da primavera ou início do verão, permitindo que as atividades de reprodução ocorram em condições ideais de temperatura, precipitação pluviométrica e umidade relativa do ar. Além das condições ambientais propícias, esta estação pode significar abundância de oferta de alimento, já que coincide com a estação das flores e da reprodução de muitos animais.

Melolonthidae sp. 1 foi registrada em alta densidade populacional em outubro e novembro e praticamente desaparece nos meses subsequentes. Este comportamento pode indicar período de reprodução da espécie. A família é conhecida por ter apenas uma geração anual e passar a fase larval no solo, no período de inverno.

Galante \& STEBNiCKa (1994), em um estudo no norte da Espanha, coletaram maior número de espécies de Aphodiidae no verão. TerRon et al. (1991), analisando a coleopterofauna da Reserva da Biosfera "La Michilia" no México em armadilhas iscadas, obtiveram maior quantidade do gênero Aphodius também no verão. Neste experimento, $A$. lividus mostrou maior atividade no outono, divergindo dos resultados observados na Espanha e no México, todavia os outros gêneros da família Aphodiidae foram mais coletados nos meses de março, outubro e novembro, ou seja, verão e primavera.

As maiores capturas também coincidiram com o período chuvoso. Dados bibliográficos tentam correlacionar a atividade destes insetos com as variáveis ambientais. Parece que a maior densidade populacional está mesmo relacionada ao conjunto de fatores que garantam a reprodução e desenvolvimento pósembrionário destes besouros. FlechtMAnn et al. (1995), comparando métodos de coleta de Scarabaeidae, verificaram que houve maior captura na armadilha luminosa na estação chuvosa. De acordo com o regime hídrico, este autor divide o ano em duas estações, uma 
chuvosa (janeiro a abril e outubro a dezembro) e uma seca (maio a setembro). Esta classificação aplica-se ao obtido nesta pesquisa. Este comportamento pode estar influenciado por fatores genéticos e é desencadeado por estímulos externos.

Kato et al. (1995), ao estudarem a sazonalidade de comunidades de insetos atraídos por armadilha de luz na Malásia durante treze meses, observaram que os Scarabaeidae coletados mostraram-se mais abundantes na estação úmida, sendo mais coletados nos meses de março, abril e dezembro. KATO et al. (2000) monitoraram quatro espécies de Melolonthidae na Malásia durante seis anos, verificando um claro padrão sazonal em três espécies. Não apresentaram significativa correlação com precipitação e o pico de captura ocorreu entre março e maio, sendo praticamente zero nos demais meses, sugerindo um ciclo de vida anual para o grupo. Situação semelhante verificou-se neste trabalho: o pico de Melolonthidae sp. 1 ocorreu entre os meses de outubro e dezembro e praticamente desapareceu nos demais meses. Todavia, NATH \& SING (1994), analisando o impacto de fatores climáticos sobre a captura de Scarabaeidae em armadilha luminosa, também coletaram os gêneros Anomala e Aphodius e ambos mostraram correlação positiva com as chuvas e negativa para temperatura e umidade relativa.

Comparativamente a outras pesquisas, utilizandose diferentes métodos de coleta, a captura de 67 espécies representa uma amostra da escarabeoideofauna local, todavia outros métodos de coleta poderiam enriquecer a lista de espécies para a região. RoDRigues et al. (2001), estudando áreas de pastagem em Aquidauana, MS e Piracicaba, SP utilizando armadilha pitfall iscadas com massa fecal fresca de bovinos, coletaram 1.767 espécimes de Scarabaeidae coprófagos pertencentes a 15 espécies para o primeiro local e 2.881 espécimes de 7 espécies para o segundo. Coletaram A. lividus, Aphodius nigrita (Fabricius, 1792), Dichotomius bos (Blanchard, 1843), Ontherus sulcator (Fabricius, 1775), Ontherus appendiculatus (Mannerheim, 1829), espécies também coletadas neste experimento, além de três morfoespécies de Ataenius não identificadas. No presente trabalho, foram capturados 1.422 espécimes coprófagos distribuídos em 25 espécies, o que mostra que a armadilha de luz também pode ser usada no estudo de besouros com este hábito.

MedRi \& Lopes (2001b), em estudo comparativo de escarabeídeos coprófagos entre área de floresta primária e pastagem adjacente, com armadilha de solo iscada, constataram que os gêneros Aphodius e Ontherus ocorreram exclusivamente na floresta, sendo que neste trabalho estes mesmos gêneros foram coletados em área antrópica, estando as armadilhas próximas ao domicílio rural e circundadas por pastagem.

Melolonthidae sp. 1 e $A$. lividus foram as duas espécies predominantes nas coletas. Segundo ODum (1986), do número total de espécies pertencentes a uma comunidade como um todo, uma percentagem relativamente pequena é abundante ou dominante, representada por grandes números de indivíduos, e uma grande percentagem é rara. ODUM (1986) afirma ainda que o padrão de umas poucas espécies comuns ou dominantes possuidoras de grandes números de indivíduos associadas com muitas espécies raras possuidoras de poucos indivíduos é característico da estrutura das comunidades nas latitudes setentrionais e nos trópicos de estações com secas e úmidas definidas.

HaLFFTER (1991) apontou que as áreas de pastagem da América Tropical apresentam menor diversidade de espécies de Scarabaeidae do que as áreas de floresta. MEdRi \& Lopes (2001b) também obtiveram maior diversidade de escarabeídeos na área florestada $\left(\mathrm{H}^{\prime}=\right.$ $2,44)$ que na área de pastagem $\left(H^{\prime}=1,75\right)$, um valor muito semelhante ao encontrado neste trabalho $\left(\mathrm{H}^{\prime}=1,74\right)$, todavia com diversidade menor naquela pesquisa e com apenas seis gêneros em comum. Os autores concluíram que a fragmentação dos hábitats naturais pode induzir mudanças na abundância e na riqueza de espécies de escarabeídeos e conseqüentemente na atuação destes organismos nos ecossistemas. Constataram ainda que estes ambientes diferem mais na composição do que no número de espécies de escarabeídeos. O estudo mostrou que a floresta possui maior diversidade do que a área de pastagem e os autores atribuíram essa diferença à alta disponibilidade de recursos alimentares como frutos, carcaças e excrementos da fauna silvestre em detrimento à quase exclusiva oferta de esterco de ruminantes pelo pasto.

$\mathrm{O}$ fato de terem sido coletadas 25 espécies $(1.422$ espécimes) de insetos com hábito detritívoro, normalmente estudadas com armadilha pitfall iscadas, é um indicativo da propriedade da armadilha de luz para coletar escarabeóideos com este hábito alimentar.

A utilização de duas armadilhas permitiu perceber a influência de área de contraste na interceptação de raios luminosos, o que pode ter influenciado na produtividade individual do equipamento. Estudos populacionais utilizando-se deste tipo de armadilha devem-se valer de repetições para evitar insucesso ou conclusões errôneas em relação a estudos populacionais. SouTHWOOD (1978) considera que a eficácia da armadilha luminosa varia de local para local, tornando-se maior quando ocorre maior contraste entre a fonte luminosa e seus arredores; talvez por este motivo a armadilha um tenha sido mais eficiente neste trabalho, pois encontrava-se em um local sem influência de outras fontes luminosas artificiais e em ambiente que facilitava a propagação da luz, com menos obstáculos ou barreiras.

Agradecimentos. Ao CNPq pela concessão de Bolsa PIBIC/CNPq e ao Dr. Fernando Z. Vaz-de-Mello pela identificação das espécies de Scarabaeoidea.

\section{REFERÊNCIAS BIBLIOGRÁFICAS}

Воотн, R. G.; Cox, M. L. \& Madge, R. B. 1990. IIE Guides to insects of importance to man. 3. Coleoptera. London, International Institute of Entomology. 384p.

Cividanes, F. J.; Neto, S. S. \& Botelho, P. S. M. 1980. Flutuação populacional de elaterídeos coletados com armadilhas luminosas em regiões canavieiras de São Paulo. Científica 8(1/2):113-119.

EMBRAPA. 1984. Levantamento de reconhecimento dos solos do Estado do Paraná. Londrina, EMBRAPA-SNLCS SUDESUL / IAPAR. 791p. (Boletim de Pesquisa, 27; IAPAR. Boletim Tecnico, 16).

Flechtmann, C. A. H.; Rodrigues, S. R. \& Couto, H. T. Z. 1995. Controle biológico da mosca-dos-chifres (Haematobia irritans 
irritans) em Selvíria, Mato Grosso do Sul. 4. Comparação entre métodos de coleta de besouros coprófagos (Scarabaeidae) Revista Brasileira de Entomologia 39(2):259-276.

Galante, E. \& Stebnicka, Z. 1994. Biogeography of Aphodiinae from the phytogeographic Orocantabrica Province, Cantabrian Range, Spain (Col. Scarabaeoidea). Acta Zoologica Cracoviensia 37(2):39-58.

HalfFTER, G. 1991. Historical and ecological factors determining the geographical distribuition of beetles (Coleoptera: Scarabaeidae: Scarabaeinae). Folia Entomologica Mexicana 82:195-238.

Halffter, G. \& Edmonds, W. D. 1982. The nesting behavior of dung beetles (Scarabaeinae) An ecological end evolutive approach. Mexico, Instituto de Ecología. 177p. (Publication, 10).

Hammer, O.; Harper, D. A. \& Ryan, P. D. 2004. Past Paleontolological statistics, version 1.28. Disponível em: $<$ http://www.folk.uio.no/ohammer/past $>$. Acesso em:12.11.2004.

Kato, M.; Itioka, T; Sakai, S; Momose, K; Yamane, S; Hamid, A. A. \& INOUE, T. 2000. Various population fluctuation patterns of light-attracted beetles in a tropical lowland dipterocarp forest in Sarawak. Population Ecology 42:97-104.

Kato, M; Inoue, T; Hamid, A.; Nagamitsu, T.; Merdek, M. B.; Nona, A. R.; Itino, T.; Yamane, S. \& Yumoto, T. 1995. Seasonality and vertical structure of light-attracted insect communities in a Dipterocarp Forest in Sarawak. Researches on Population Ecology 37(1):59-79.

MAACK, R. 1981. Geografia física do Estado do Paraná. 2.ed. Rio de Janeiro, José Olympio. 450p.
Marinoni, R. C. \& Dutra, R. R. 1991. Levantamento da fauna entomológica no estado do Paraná. I. Introdução. Situações climáticas e florísticas de oito pontos de coleta. Dados faunísticos de agosto de 1986 a julho de 1987. Revista Brasileira de Zoologia 8(1-4):31-73.

Medri, I. \& Lopes, J. 2001a. Coleopterofauna em floresta e pastagem no norte do Paraná, Brasil, coletada com armadilha de solo. Revista Brasileira de Zoologia 18(Supl.1):125-133.

2001b. Scarabaeidae (Coleoptera) do Parque Estadual Mata dos Godoy e de área de pastagem, no norte do Paraná, Brasil. Revista Brasileira de Zoologia 18(Supl.1):135-141.

NAth, P. \& Singh, J. 1994. Impact of weather factors on lighttrap catches of scarabaeid beetles (Col., Scarabaeidae). GIENDG 7:137-141.

Odum, E. P. 1986. Ecologia. Rio de Janeiro, Guanabara. 434p. Rodrigues, S. R.; Marchini, C. \& Mendes, C. C. 2001. Ácaros da família Macrochelidae (Gamasida) associados com besouros coprófagos (Scarabaeidae). Revista Brasileira de Entomologia 45(3):207-214.

Southwood, T. R. E. 1978. Ecological methods: with particular reference to the study of insect populations. London, Chapman and Hall. 524p.

Terron, R. A.; Anduaga, S. \& Moron, M. G. 1991. Analisis de la coleopterofauna necrofila de la Reserva de La Biosfera "La Michilia", Durango, México. Folia Entomologica Mexicana 81:315-324.

URBAn, T. 1996. Nossas árvores. Manual para recuperação da reserva florestal legal. Sociedade de Pesquisa em Vida Selvagem e Educação Ambiental. Curitiba, FNMA. 86p.

Recebido em agosto de 2004. Aceito em dezembro de 2005. ISSN 0073-4721

Artigo disponível em: www.scielo.br/isz 